\title{
Modelamiento Predictivo de la Pérdida Auditiva Laboral, Relacionada con el Tratamiento de Absorción Acústica en una Industria Metal-Mecánica en Chile
}

\section{PREDICTIVE MODELING OF LABOR HEARING LOSS, RELATED TO THE TREATMENT OF ACOUSTIC ABSORPTION IN METAL-MECHANIC INDUSTRY IN CHILE}

\author{
Aldo Esteban Canales Montenegro', Aldo Mauricio Campos Pérez², José Luis Cárdenas Bergmann ${ }^{3}$ \\ 1. Ingeniero en Sonido y Acústica. Diplomado en Prevención de Riesgos, Medio Ambiente, Salud Ocupacional e Higiene Industrial. \\ 2. Ingeniero Acústico, Magister en Ingenieria Estructural y Geotécnica. \\ 3. Académico Universidad de Chile, Ingeniero de Ejecución en Sonido, Magíster en Educación.
}

\begin{abstract}
RESUMEN
La presente investigación evalúa el impacto que tiene en la protección de la audición de los trabajadores, la incorporación del tratamiento acústico del entorno laboral correspondiente al sector metal-mecánico de la industria Inchalam, ubicada en Santiago de Chile, cuyo entorno es tratado con materiales absorbentes certificados, ubicados estratégicamente en las zonas de mayor exposición al ruido en dicha industria, considerado este sector productivo como el más vulnerable en cuanto a patologías auditivas. Los resultados de la investigación evidencian que diversos escenarios propuestos para el tratamiento acústico absorbente pueden llegar a valores de reducción máximos de $2 \mathrm{~dB}$ en la exposición a ruido de algunos puestos de trabajo, concluyéndose que es una alternativa efectiva en términos de reducción de la exposición ocupacional a ruido de los trabajadores, cuando la actividad laboral y maquinarias no permite tratar acústicamente la fuente ni el receptor. Respecto de la proyección de la Pérdida Auditiva, según los criterios de la Norma IS01999 (Determination of occupational noise exposure and estimation of noise induced hearing impairment) y Prexor (Protocolo de Exposición Ocupacional al Ruido), se modeló una población de trabajadores varones de 50 años de edad y 30 años de exposición al ruido. La configuración diseñada permite predecir una disminución de hasta $5 \mathrm{~dB}$ en la banda más crítica, lo que implica una disminución de $3 \mathrm{~dB}$ promedio en la curva audiométrica, que a su vez incide en el 50\% de disminución de la energía acústica recibida en 30 años de exposición y que para el caso estudiado aleja a esta población de un daño médico-legal y con ello de las eventuales indemnizaciones.
\end{abstract}

(Canales A, Campos A, Cárdenas J, 2016. Modelamiento Predictivo de la Pérdida Auditiva Laboral, Relacionada con el Tratamiento de Absorción Acústica en una Industria Metal-Mecánica en Chile. Cienc Trab. May-Ago; 18 [56]: 73-80).

Palabras claves: HIPOACUSIA, ISO 1099, ACÚSTICA, PREXOR, EXPOSICIÓN AL RUIDO, DOSIS DE RUIDO, PÉRDIDA AUDITIVA, AUDIOMETRÍA, ENFERMEDAD OCUPACIONAL.

\section{ABSTRACT}

The investigation will assess the impact on hearing protection for workers, a metal mechanic sector Inchalam industry located in Santiago of Chile, the incorporation of acoustic treatment of the working environment, with certified absorbent materials, strategically located in areas greater exposure to noise in the industry. The project includes the use and acquisition of acoustic software RapOne a Soft-dB Canadian Company, that allows input measurements Sound Pressure Level Equivalent directly to software from the activity measured. The results of the research conducted show that various scenarios proposed for the absorbent acoustic treatment, can reach maximum values of $2 \mathrm{~dB}$ reduction in noise exposure of some jobs, concluding that it is an effective alternative in terms of reduction occupational noise exposure of workers when the work activity and machinery does not allow acoustically treat to the source or receiver. About the projection of Hearing Loss, according to the criteria of the Standard ISO1999 and Prexor, a population of male workers 50 years old and 30 years working, was modeled noise exposure, the configuration modeled to predict a decrease of up to 5 $\mathrm{dB}$ in the most critical band, which implies a decrease of $3 \mathrm{~dB}$ average audiometric curve, corresponding to 50\% decrease of the acoustic energy received by 30 years of exposure and for the case studied this population away from harm legal medical and thus of any indemnities.

Keywords: HEARING LOSS, ACOUSTICS, DOSE NOISE, NOISE EXPOSURE, AUDIOMETRIC, OCCUPATIONAL DISEASE.

\section{INTRODUCCIÓN}

La Hipoacusia Neurosensorial de origen laboral se deriva de la exposición al ruido industrial o por trauma acústico, lo que produce daño

Correspondencia / Correspondence: Aldo Esteban Canales Montenegro e-mail: aldo.canales.m@gmail.com Recibido: 21 de Enero de 2016 / Aceptado: 03 de Marzo de 2016 irreversible en trabajadores expuestos a ruido sobre los 85 decibeles en una jornada diaria de ocho horas. ${ }^{1}$ El perfil de quienes la sufren son principalmente hombres de entre 50 y 65 años, que trabajan cerca de fuentes de ruido con elevados niveles de presión sonora. La Hipoacusia es la enfermedad ocupacional de mayor prevalencia en nuestro país; de hecho, según el Sistema Automatizado de Información en Salud Ocupacional (SAISO) del Instituto de Salud Pública, entre los años 1997 y 2000, el primer lugar de los diagnósticos de ingreso corresponde a las enfermedades del oído, con un 38\%. ${ }^{2}$ Para generar un aporte en la disminución y/o control de la Hipoacusia Neurosensorial Laboral por actividades laborales, el presente proyecto 
Figura 1.

Porcentaje de Hipoacusia Neurosensorial por actividad productiva. (Fuente ACHS).

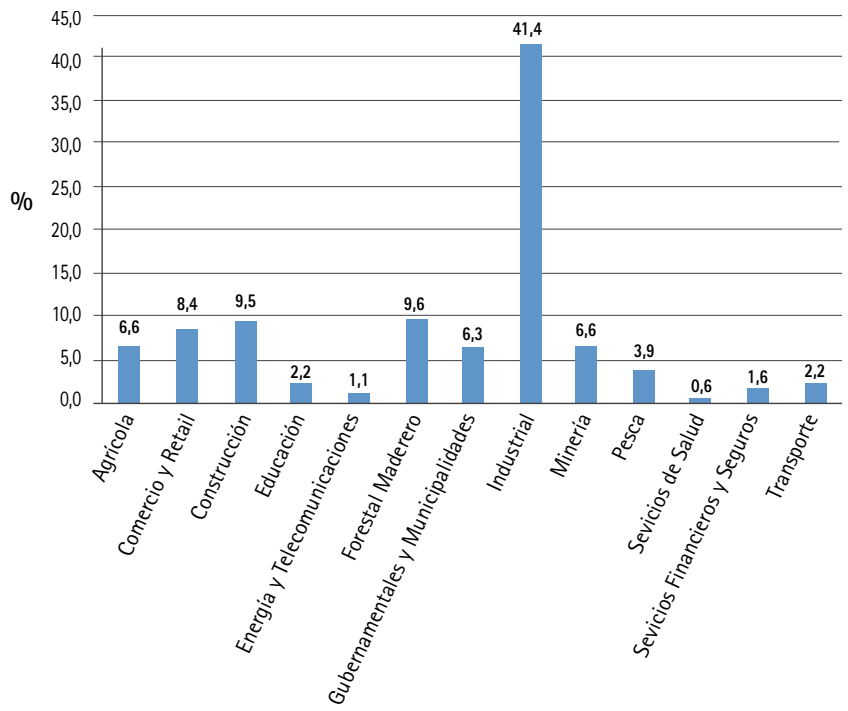

propone evaluar de forma cuantitativa la influencia en la exposición ocupacional a ruido, mediante la incorporación de tratamiento absortor acústico del entorno laboral, con materiales porosos ubicados al interior de los lugares de trabajo.

La alternativa propuesta en esta investigación, de lograr una reducción de ruido mediante absorbentes acústicos, es relevante al momento de contemplar ciertas faenas, máquinas y situaciones laborales donde no es posible aplicar la reducción de ruido por encierros acústicos, ni protectores auditivos y, por lo tanto, la situación planteada es una alternativa efectiva en términos de la exposición ocupacional a ruido de los trabajadores. Además, hay que considerar que los paneles acústicos instalados en las inmediaciones de la fuente reducen la distancia crítica de la fuente. ${ }^{3}$ Este modelo base, o de condición inicial, será calibrado en referencia a las anteriores y nuevas mediciones, comparando los niveles de ruido equivalentes (LAeq) y Dosis de Ruido de cada puesto de trabajo. Lo anterior tiene como marco normativo lo indicado en el Prexor, Decreto Supremo 594 de Chile y la Norma Internacional IS01999. Las mediciones fueron realizadas entre los años 2012 y 2014 en situaciones homogéneas de la actividad laboral de dicha industria, correspondiente a la sección de mallas Electrosoldadas de la Industria Inchalam, una de las mayores empresas de fabricación de mallas y alambres en Chile. Posteriormente se diseñó un modelo matemático a partir de la condición inicial, que incorporó distintas configuraciones de tratamiento

Figura 2.

Sección Mallas Electrosoldadas.

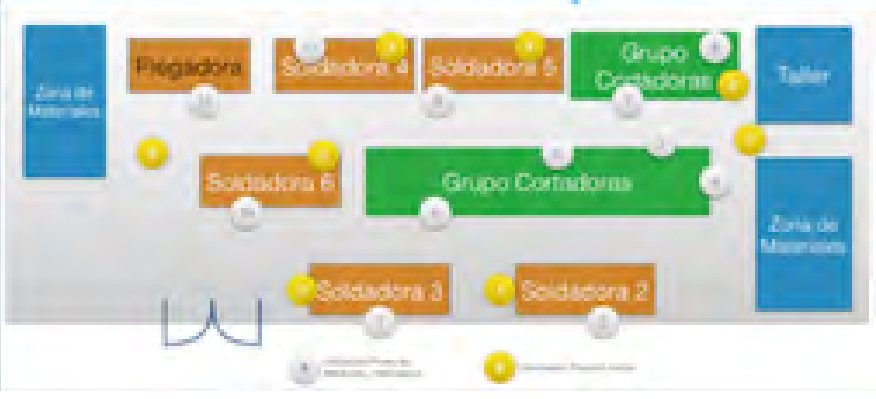

acústico con materiales absorbentes en las superficies interiores del lugar de trabajo, donde se simula el comportamiento real del contaminante acústico al interior del recinto, por medio del mapa de ruido que entrega el software Rap-One. Esta herramienta permite visualizar los puntos sensibles de exposición, y de esta forma graficar la propagación del ruido desde las fuentes emisoras hasta los puntos de recepción, es decir, los trabajadores, tomando en consideración el aporte originado por el sonido directo y por el campo reverberante de la sala producido por las propiedades acústicas de cada elemento al interior del recinto.

\section{MATERIAL Y MÉTODOS}

Con el objetivo de evaluar y determinar la exposición de los trabajadores y el nivel de riesgo que le implican sus respectivos puestos de trabajo, se realizan mediciones mediante dosímetro a cada uno de los trabajadores considerados los más expuestos y/o representativos en términos de exposición a ruido dentro de su grupo homogéneo, en los horarios definidos como de mayor demanda y durante un periodo considerado representativo de su jornada laboral habitual. Paralelamente se realizan mediciones ambientales en cada fuente de ruido con el objeto de evaluar la eventual exposición más crítica de los trabajadores a la fuente. Todas las mediciones se realizaron con ponderación en las curvas A y $\mathrm{C}$.

En el protocolo Prexor ${ }^{4}$ se establecen Niveles de Riesgo con el objetivo de calificar la exposición ocupacional a ruido, para que las empresas gestionen las acciones de control, como también la vigilancia de la salud de sus trabajadores expuestos.

Figura 3.

Condición, Calificación y Acción según Niveles de Riesgo.

\begin{tabular}{|c|c|c|c|}
\hline $\begin{array}{l}\text { Nivel } \\
\text { de Riesgo }\end{array}$ & Condición & Calificación & Acción \\
\hline 1 & Dosis $<0,5$ & Aceptable & $\begin{array}{l}\text { Mantener o mejorar condiciones de } \\
\text { exposición. La evaluación tendrá una } \\
\text { periodicidad de } 3 \text { años. }\end{array}$ \\
\hline 2 & $\begin{array}{c}0,5 \leq \text { Dosis } \\
<10 \mathrm{DMP}\end{array}$ & Importante & $\begin{array}{l}\text { Plazo máximo de } 1 \text { año para desarrollar } \\
\text { medidas de control eficaces. A partir de } \\
\text { este nivel los trabajadores son } \\
\text { ingresados a programa de vigilancia médica. }\end{array}$ \\
\hline 3 & Dosis $\geq 10 \mathrm{DMP}$ & Critica & $\begin{array}{l}\text { Plazo máximo de } 6 \text { meses para desarrollar } \\
\text { medidas de control eficaces. A partir de } \\
\text { este nivel los trabajadores son ingresados } \\
\text { a programa de vigilancia médica. }\end{array}$ \\
\hline
\end{tabular}

\section{Los Niveles de Riesgo se definen a continuación}

La metodología del estudio corresponde, en una primera parte, a Investigación de Campo, donde se recopilan los antecedentes laborales, turnos, jornadas, planimetría, maquinaria, operarios y tipos de trabajos de la sección de mallas Electrosoldadas de la empresa Inchalam (www.inchalam.bekaert.com), y, en una segunda parte, se procede a una Investigación Cuantitativa, aplicando mediciones objetivas de NPS, Dosis y modelos computacionales acorde a la normativa chilena, referida al D.S. 594/99 y Prexor. ${ }^{3,5-7}$

El plan de trabajo a desarrollar contempla los siguientes procedimientos:

a. Determinar y seleccionar la condición acústica del recinto representativo para el estudio.

b. Modelar teóricamente el campo sonoro en los recintos seleccionados aplicando el software de la empresa Soft-dB para modelar las condiciones. 
c. Estimar teóricamente las variaciones en las exposiciones a ruido al modificar los parámetros acústicos del recinto.

d. Entregar lineamientos cuantitativos en relación al impacto del acondicionamiento acústico de un recinto en la exposición a ruido ocupacional.

e. Proyectar la conservación de la audición de trabajadores en la situación planteada, de acuerdo a la normativa ISO $1999 .{ }^{8}$

La población sociodemográfica a estudiar corresponde a 27 trabajadores de la ciudad de Santiago de Chile, cuyo rango etario se encuentra entre los 30 y 55 años; trabajadores que desempeñan variadas actividades en un área efectiva de $1000 \mathrm{~m}^{2}$ correspondiente al sector denominado Mallas Electrosoldadas. Es un recinto cerrado, con paredes laterales de zinc, sin muros divisorios en su interior, y con algunos paneles difusores de ruido para algunas máquinas, cuyo funcionamiento es electro-mecánico. Este sector se considera significativo por cuanto el ruido es un riesgo evidente para las actividades ocupacionales.

A partir de datos estadísticos sobre enfermos de Hipoacusia Neurosensorial Laboral, por cada actividad productiva se seleccionan escenarios representativos de mayor riesgo auditivo para el estudio. ${ }^{9}$

Tabla 1.

Condición, Calificación y Acción según Niveles de Riesgo.

\begin{tabular}{|c|c|c|c|c|c|c|}
\hline Área & $\begin{array}{l}\text { Puesto de } \\
\text { Trabajo }\end{array}$ & Función & $\begin{array}{l}\text { Tiempo } \\
\text { De Exposición } \\
\text { (Te) Hrs. }\end{array}$ & Turno 1 & $\begin{array}{l}\text { Grupo } \\
\text { Homogéne } \\
1 \text { Turno } 2\end{array}$ & $\begin{array}{l}\text { o } \\
\text { Turno } 3\end{array}$ \\
\hline \multirow{5}{*}{$\begin{array}{l}\text { Planta } \\
\text { Mallas } \\
\text { Electro- } \\
\text { soldadas }\end{array}$} & $\begin{array}{l}\text { Operador } \\
\text { Cortadora }\end{array}$ & $\begin{array}{l}\text { Monitoreo, control } \\
\text { y mantenimiento } \\
\text { de máquina }\end{array}$ & 7,5 & 2 & 2 & 2 \\
\hline & $\begin{array}{l}\text { Operador } \\
\text { Soldadora }\end{array}$ & $\begin{array}{l}\text { Monitoreo, control } \\
\text { y mantenimiento } \\
\text { de máquina }\end{array}$ & 7,5 & 8 & 5 & 4 \\
\hline & $\begin{array}{l}\text { Operador } \\
\text { Puente Grúa }\end{array}$ & $\begin{array}{c}\text { Control maquinaria, } \\
\text { carga y descarga } \\
\text { de elementos }\end{array}$ & 7,5 & 1 & 1 & 1 \\
\hline & $\begin{array}{l}\text { Operador } \\
\text { Grúa } \\
\text { Horquilla }\end{array}$ & $\begin{array}{c}\text { Carga y } \\
\text { descarga } \\
\text { de productos }\end{array}$ & 7,5 & 1 & - & - \\
\hline & \multicolumn{3}{|c|}{ Total de trabajadores en la sección } & & 27 & \\
\hline
\end{tabular}

De este análisis matemático y estadístico se definen variables como la actividad y proceso productivo, número de trabajadores, maquinarias, turnos de trabajo y fuentes de ruido relacionada.

Además se determinan los aspectos acerca de la materialidad del recinto seleccionado con sus características acústicas y geométricas, para posteriormente levantar un modelo computacional teórico sobre el software Rap-One de modelación y propagación acústica en interiores, a partir del cual se obtendrán las zonas críticas en Leq. Lw y dosis de ruido. En la figura se ejemplifica lo entregado por el software, donde resultan evidentes las zonas críticas, en que el Leq se encuentra entre 93 y $95 \mathrm{~dB}$, coloreadas en rojo y naranjo, según el indicador.

\section{INSTRUMENTACIÓN}

Para el desarrollo de la investigación se utilizan los siguientes instrumentos acústicos certificados:

- Software RAP-One diseñado por la Empresa Softdb. Este instrumento tiene la particularidad de adquirir y asignar directamente desde el sonómetro la lectura de los puntos críticos asociados a

Figura 6.

Dosimetro, Sonómetro y Calibradores.

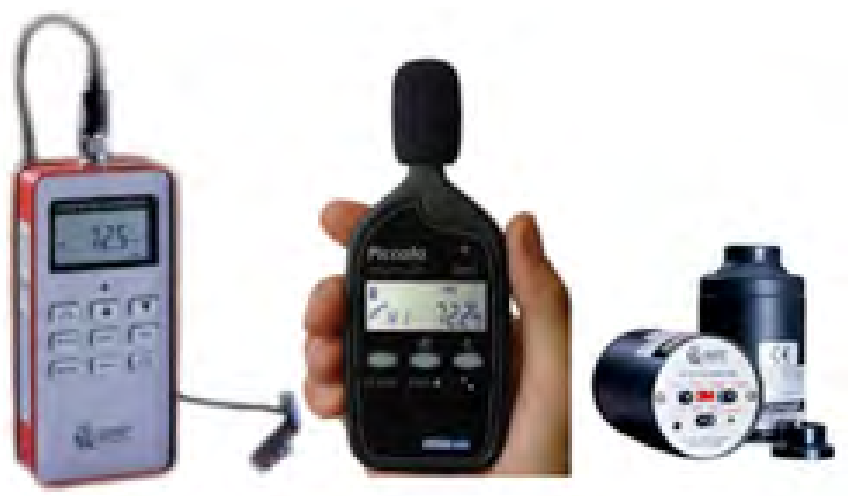

Figura 5. Mapa de Ruido Experimental.

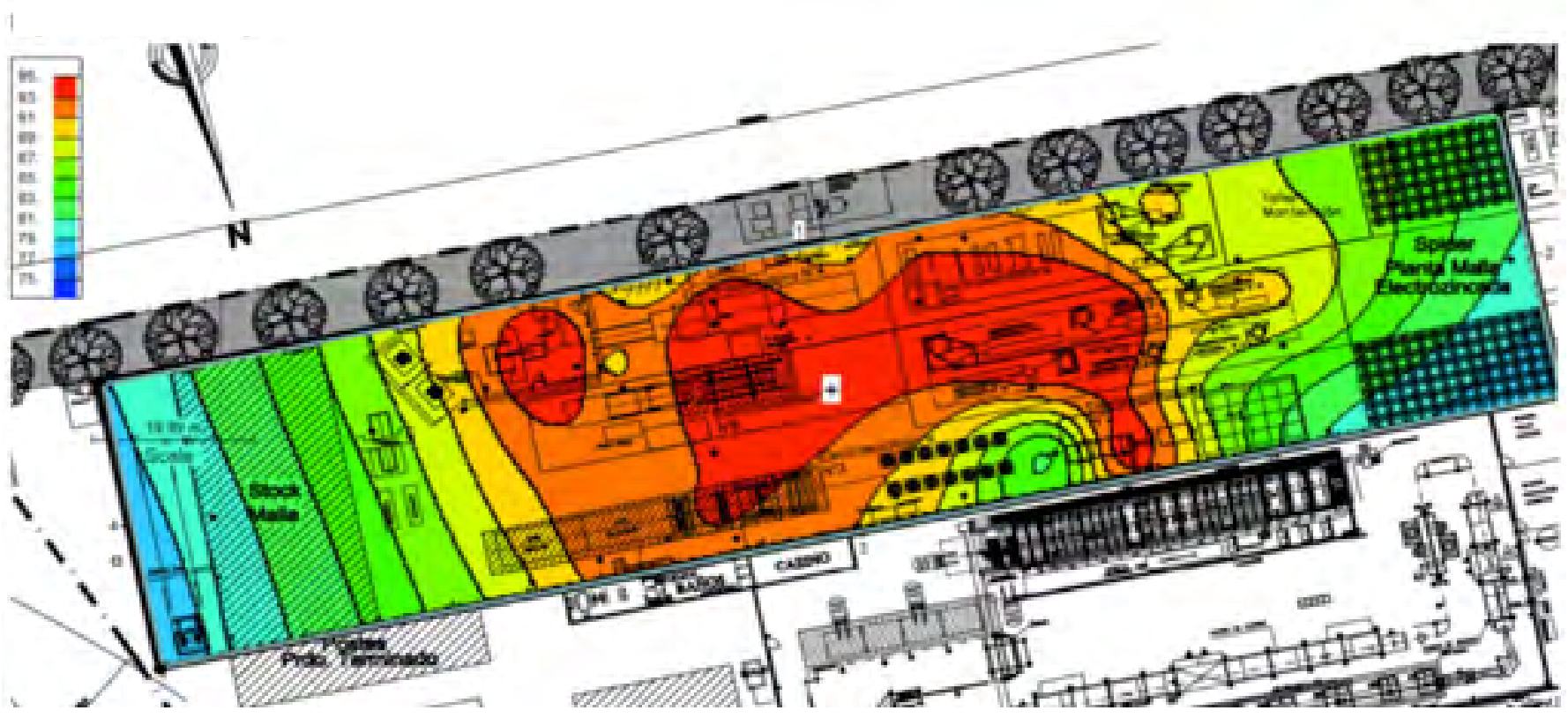


un mapa predeterminado. Esta simpleza en la adquisición y tabulación de datos permite una mayor recogida de puntos de medición en menor tiempo de análisis. http://www.softdb.com/fr/ index.php

- Sonómetro Piccolo: Modelo SLM-P3, correspondiente a un sonómetro integrador y datalogger de Tipo 2, que además cuenta con la característica de conectarse con el Software Rap-One y en conjunto generar un mapa de ruido en tiempo real denominado Mapa Experimental.

- Calibrador Acústico Quest modelo QC-10 certificado por el Laboratorio ACHS y Calibrador Acústico Sonómetro Softdb TM-100.

- Dosímetro Quest: Modelo Q400, instrumento calibrado en Laboratorio ACHS.

\section{RESULTADOS}

Se obtuvo el Nivel de Presión Sonora en 40 puntos asociados al sector productivo, a partir del cual 12 puntos críticos son seleccionados como representativos por el mayor riesgo de ruido para las actividades ocupacionales. Estos datos son utilizados para calibrar el modelo virtual en el software a fin de obtener los Niveles de Potencia Acústica (Lw) de cada fuente de ruido presente en el área de trabajo, correspondiente a un galpón de 1000 metros cuadrados. Una vez calculados los Lw de cada fuente, se realiza un modelo simulado del escenario real, que además se complementa con mediciones de Tiempo de Reverberación y Coeficiente de Absorción de los principales materiales constructivos presentes, considerando que ambas variables están implícitas en las iniciales mediciones de Lpeq.

El siguiente plano corresponde a la visualización de los Lpeq obtenidos en la medición de 40 puntos en la sección evaluada mediante el software Rap-One, con datos adquiridos con sonómetro de la misma empresa.

Los resultados obtenidos de la comparación entre los niveles de ruido medidos y los niveles simulados para la misma ubicación se muestran a continuación.
Tabla 2.

Comparación de resultados Validación 1.

\begin{tabular}{lccc|}
$\begin{array}{l}\text { No Punto } \\
\text { de Medición }\end{array}$ & $\begin{array}{c}\text { Medición } \\
\text { Lpeq } \mathrm{dB}(\mathrm{A})\end{array}$ & $\begin{array}{c}\text { Modelo } \\
\text { Lpeq } \mathrm{dB}(\mathrm{A})\end{array}$ & $\begin{array}{c}\text { Diferencia } \\
\mathrm{dB}(\mathrm{A})\end{array}$ \\
\hline 1 & 93,0 & 93,5 & $-0,5$ \\
2 & 93,9 & 94,4 & $-0,5$ \\
3 & 88,8 & 91,4 & $-2,6$ \\
4 & 89,3 & 89,8 & $-0,5$ \\
5 & 94,6 & 92,2 & 2,4 \\
6 & 93,0 & 92,7 & 0,3 \\
7 & 89,4 & 91,3 & $-1,9$ \\
8 & 89,3 & 91,0 & $-1,7$ \\
9 & 94,3 & 91,6 & 2,7 \\
10 & 93,3 & 93,8 & $-0,5$ \\
11 & 95,7 & 98,0 & $-2,3$ \\
12 & 94,4 & 95,4 & -1 \\
\hline
\end{tabular}

El segundo proceso de validación corresponde a la comparación entre los Niveles de Presión Sonora Equivalentes (Leq) de 8 puestos críticos de trabajo, lo cual se realiza mediante dosímetros marca Quest, modelo Q400, certificados por el Laboratorio de la ACHS durante un periodo representativo de la jornada de trabajo de cada trabajador, versus los niveles Leq simulados con el software Rap-One en el escenario anteriormente validado.

Tabla 3.

Resultados Validación 2: Dosimetrías.

\begin{tabular}{|ccccc} 
№ & Puesto De Trabajo & $\begin{array}{c}\text { Medición } \\
\text { Leq dB(A) }\end{array}$ & $\begin{array}{c}\text { Simulación } \\
\text { Leq dB(A) }\end{array}$ & $\begin{array}{c}\text { Diferencia } \\
\mathrm{dB}(\mathrm{A})\end{array}$ \\
\hline 1 & Operador Cortadora 4 & 93,3 & 93,4 & $-0,1$ \\
2 & Operador Soldadora 2 & 93,3 & 92,9 & 0,4 \\
3 & Operador Soldadora 3 & 91,4 & 91,6 & $-0,2$ \\
4 & Operador Soldadora 4 & 93,3 & 93 & 0,3 \\
5 & Operador Soldadora 5 & 93 & 92,8 & 0,2 \\
6 & Operador Soldadora 6 & 92,1 & 91,9 & 0,2 \\
7 & Operador Grúa Horquilla & 88,7 & 88,3 & 0,4 \\
8 & Operador Puente Grúa & 92,5 & 92,5 & 0
\end{tabular}

Figura 7. Mapa de Ruido Experimental.

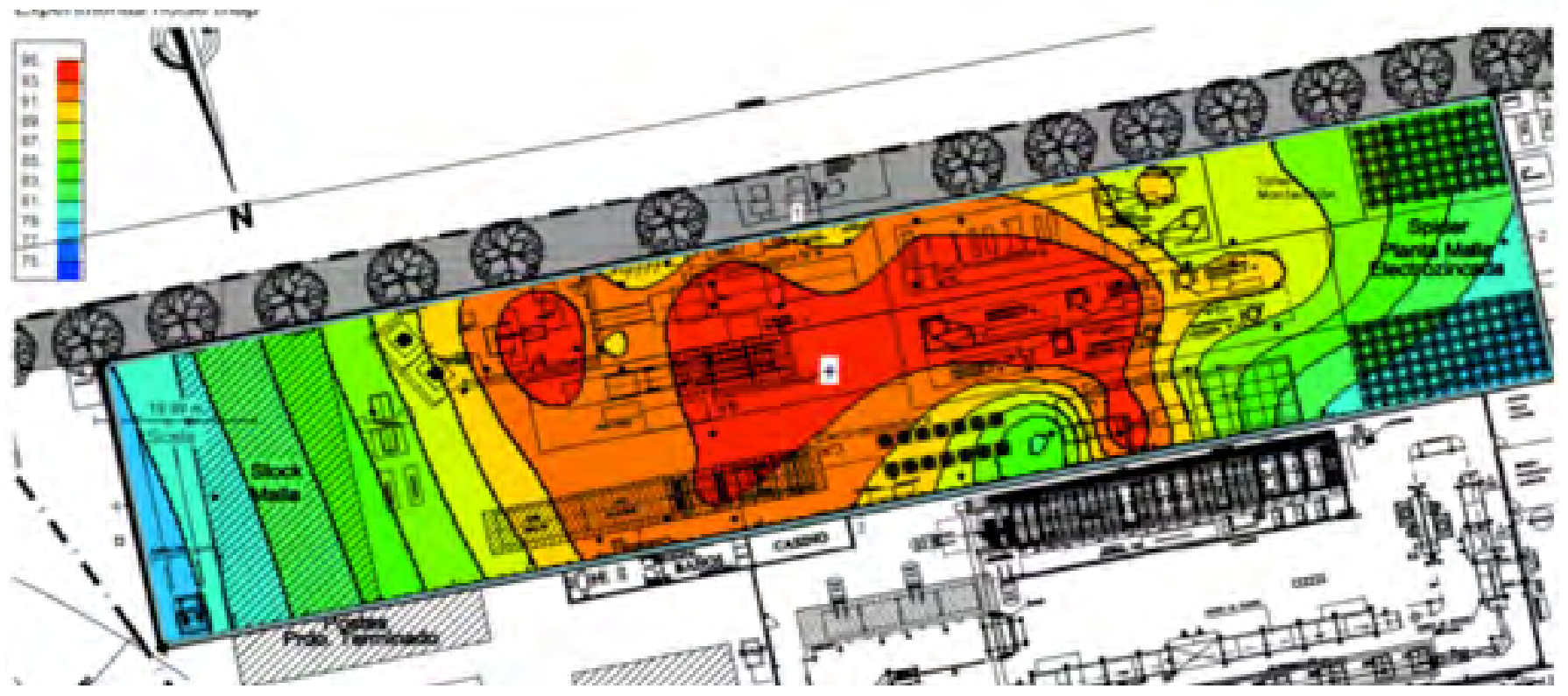


Figura 9. Mapa de Ruido de Referencia.

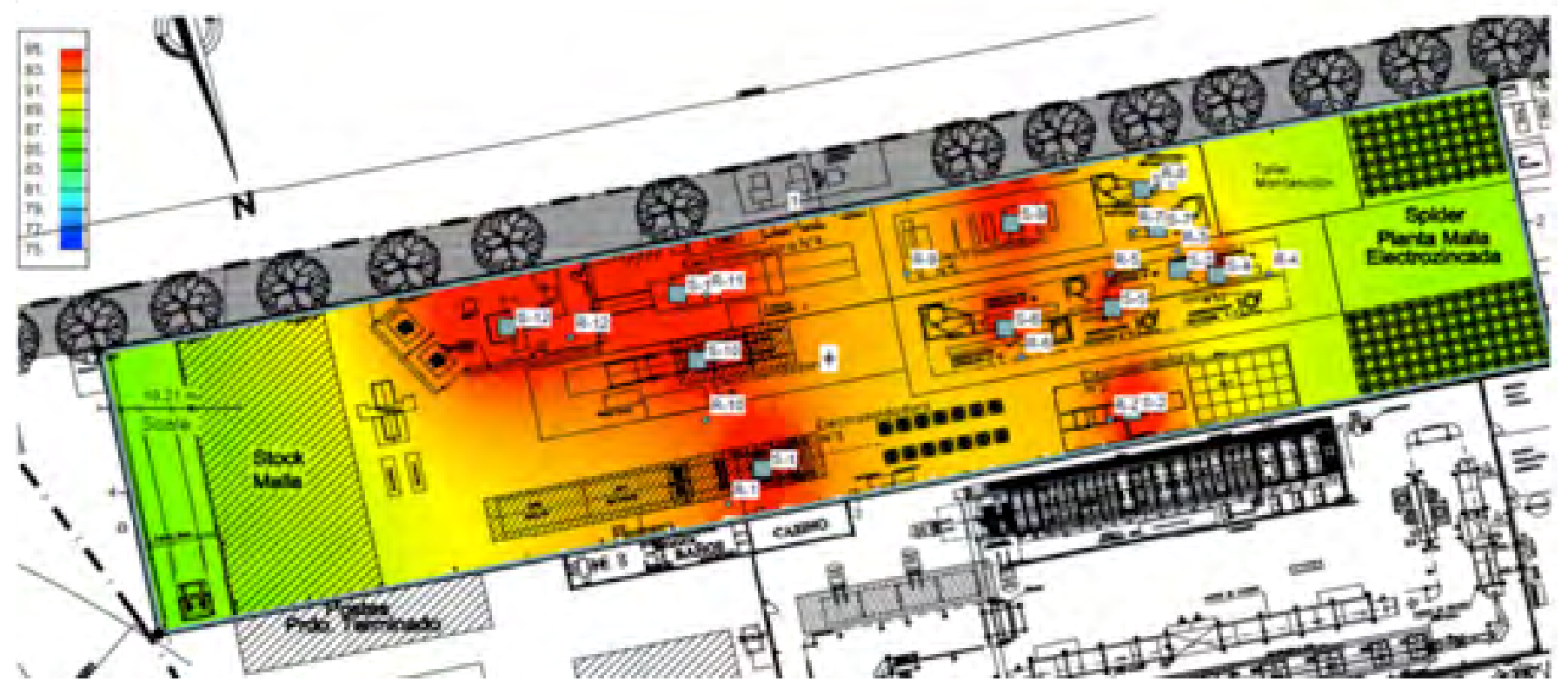

A partir de esto, se simula la condición del entorno laboral revestido con el material Absortor Acústico certificado, Hipertec Wall Sound de $50 \mathrm{~mm}$ de espesor y densidad de $100 \mathrm{~kg} / \mathrm{m}^{3}$ con el cual el software evidencia la reducción de Niveles de Exposición a ruido. Cada panel presenta dimensiones de 1x7 $\mathrm{m}$ y se ubica en los muros adyacentes a las principales fuentes de ruido. De esta forma, cada muro contempla $420 \mathrm{~m}^{2}$ de material absorbente sonoro.

Esta configuración resulta ser una de las más favorables en términos de absorción sonora y la de menor costo monetario dentro de las alternativas de superficie absorbente. Se observa en el mapa de ruido el efecto de dicho material.

Los resultados de los puestos de trabajo, en este escenario, se ven reducidos según se indica en la tabla siguiente:
Tabla 4.

Resultados Caso 1.

\begin{tabular}{|ccccc|} 
No $^{\circ}$ & Puesto De Trabajo & $\begin{array}{c}\text { Medición } \\
\text { Leq dB(A) }\end{array}$ & $\begin{array}{c}\text { Simulación } \\
\text { Leq } \mathrm{dB}(\mathrm{A})\end{array}$ & $\begin{array}{c}\text { Diferencia } \\
\mathrm{dB}\end{array}$ \\
\hline 1 & Operador Cortadora 4 & 93,3 & 91,7 & 1,7 \\
2 & Operador Soldadora 2 & 93,3 & 91,1 & 1,8 \\
3 & Operador Soldadora 3 & 91,4 & 89,8 & 1,8 \\
4 & Operador Soldadora 4 & 93,3 & 91,4 & 1,6 \\
5 & Operador Soldadora 5 & 93 & 91,1 & 1,7 \\
6 & Operador Soldadora 6 & 92,1 & 90,4 & 1,5 \\
7 & Operador Grúa Horquilla & 88,7 & 86,1 & 2,2 \\
8 & Operador Puente Grúa & 92,5 & 90,8 & 1,7 \\
\hline
\end{tabular}

Figura 11. Mapa de Ruido Caso 1.

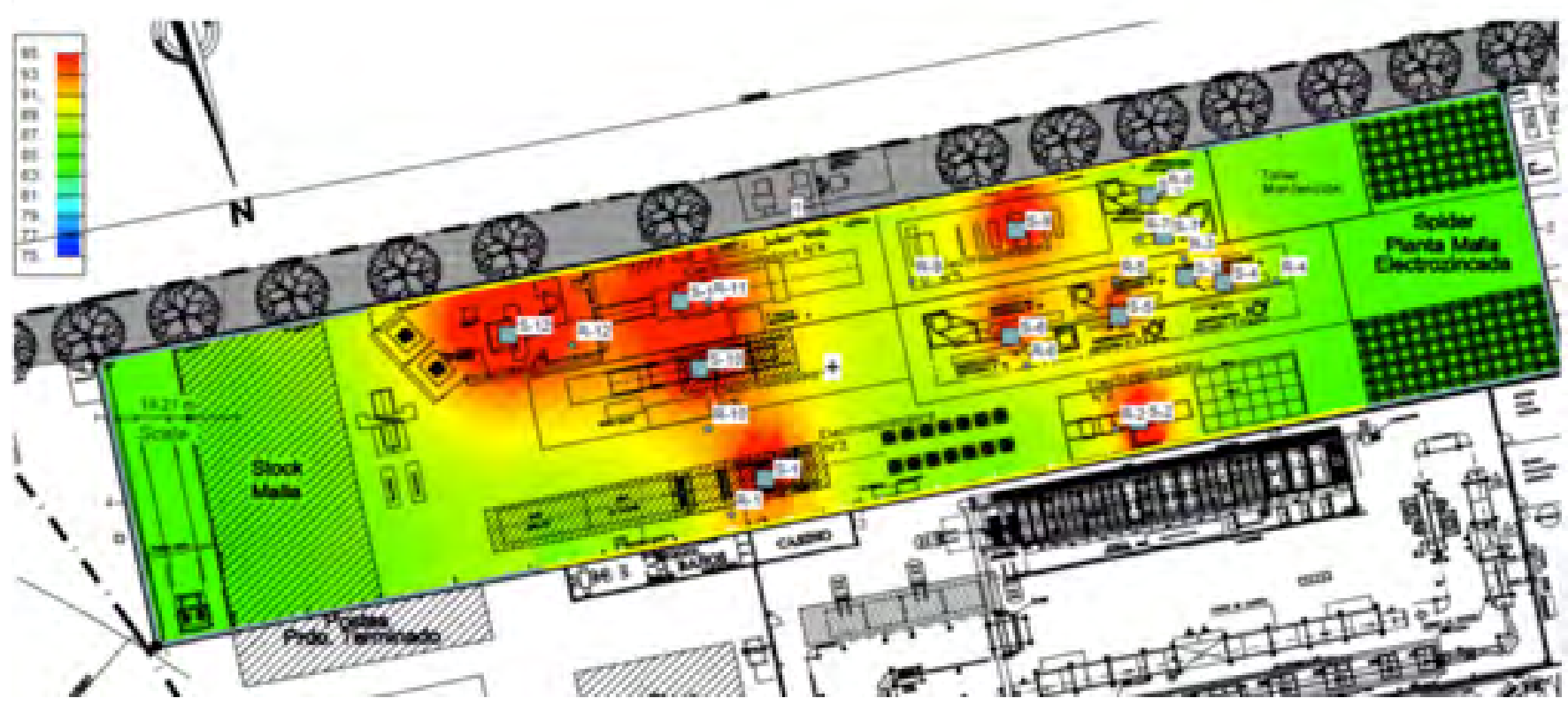


Según los resultados obtenidos de la simulación del caso mencionado, se obtiene $1,8 \mathrm{~dB}$ en promedio de reducción del nivel de exposición ocupacional a ruido en los puestos de trabajo. De la misma forma, al evaluar condiciones similares para comparar dimensiones, ubicaciones y espesor del material empleado, para observar la correspondencia en la mitigación, se puede lograr hasta 2,2 $\mathrm{dB}$ de atenuación en las mejores condiciones, pero de mayor costo económico.

En base a la norma ISO1999:1990 Acoustics: Determinations of occupational noise exposure and estimation of noise-induced hearing impairment ${ }^{8}$ se desarrolla un modelo mediante Excel para estimar y predecir el daño auditivo que podría adquirir un trabajador considerando variables etarias, nivel de exposición y años de exposición. Se concluye que en promedio se podría reducir hasta en $5 \mathrm{~dB}$ la pérdida auditiva, como se observa en los siguientes gráficos, que según la literatura corresponde al 50\% de disminución de la energía acústica recibida en 30 años de exposición, situación satisfactoria para el presente estudio.

\section{CONCLUSIONES}

A partir de la metodología e instrumentación empleada se hace posible predecir los niveles de ruido en las plantas de trabajo y con ello obtener una estimación de la exposición ocupacional a ruido en los puestos de trabajo y sus respectivas proyecciones de eventuales pérdidas auditivas en el tiempo.

Por esto, la importancia de considerar el aspecto acústico dentro de los proyectos de construcción de recintos laborales posibilita la optimización en términos de exposición a ruido de la distribución de fuentes de ruido al interior de la planta por medio del desarrollo de mapas estratégicos de ruido que permitan la comparación de resultados de diversos escenarios de configuración de la planta.

Este estudio inclusive podría ser desarrollado como una asesoría por parte de las unidades de estudio y especialidades técnicas de las mutualidades correspondientes.

Además, se indica que la instalación de sistemas de control de ruido es prioridad, y de existir imposibilidad justificada de implementación, corresponde el estudio de la ejecución de medidas administrativas, dejando en último lugar el uso de elementos de protección auditiva, debido a que no garantizan la protección auditiva del usuario. En la medida de lo posible, considerar al interior de la planta de producción la separación de áreas por medio de pantallas acústicas completas o parciales con el objeto de evitar la contribución cruzada de ruido entre maquinarias y puestos de trabajo cercanos. Por lo anterior, se recomienda implementar cerramientos acústicos considerando la factibilidad técnica y de operación para las máquinas y motores.

De acuerdo con la jerarquía definida en la norma chilena Prexor para la implementación de sistemas de control de ruido, la instalación de tratamiento acústico de absorción en recintos ocupa el segundo lugar de prioridad, siendo una medida de control en el medio de transmisión entre la fuente y el receptor.

Esta situación se confirma según los resultados obtenidos, puesto que la implementación de tratamiento acústico absorbente puede llegar a valores de reducción máximos de $2 \mathrm{~dB}$ en la exposición a ruido de algunos puestos de trabajo.

Los resultados arrojados por la evaluación de exposición laboral a ruido para los puestos de trabajo evaluados y ubicados en el área de Mallas Electrosoldadas obtuvieron una Dosis de Ruido Diaria

\section{Gráfico 1.}

Estimación de pérdida auditiva.

\section{1: Op. Cortadora 4}

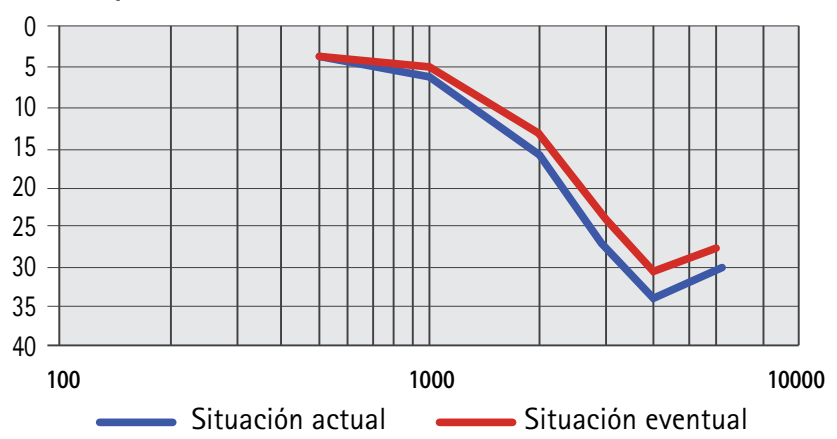

3: Op. Soldadora 3

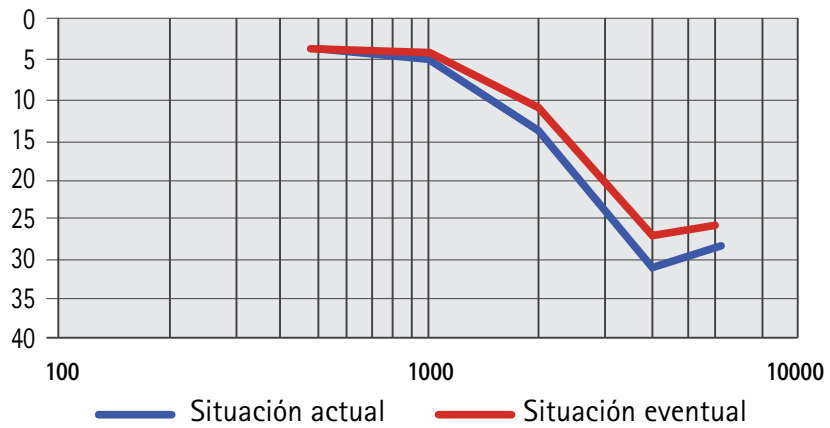

3: Op. Soldadora 3

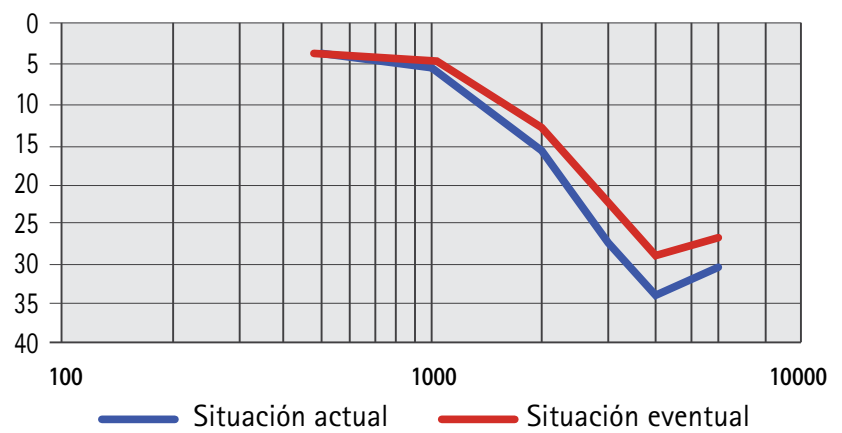

4: Op. Soldadora 4

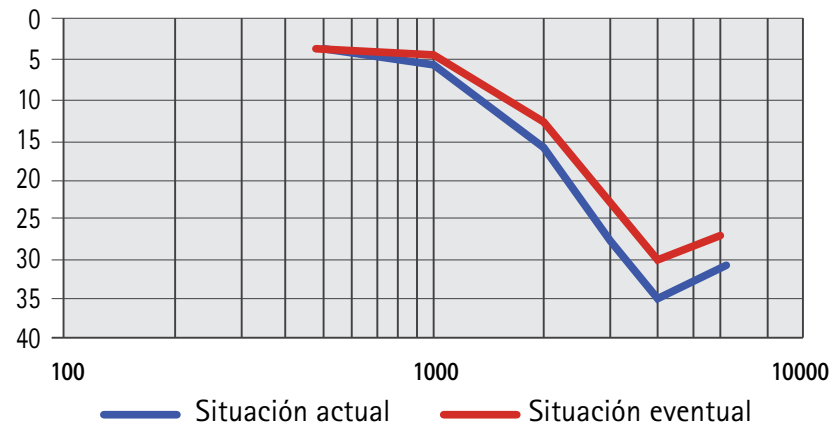


sobre el criterio preventivo establecido como Dosis de Acción (DA) en Prexor tanto para turnos diurnos y nocturnos, por lo que su clasificación de riesgo es de 2, correspondiente a una dosis Importante.

De la proyección de la Pérdida Auditiva realizada mediante IS01999, con variables definidas para hombres en promedio de 50 años de edad y proyectada a 30 años de exposición al ruido, entre el escenario actual y un escenario eventual que considera la configuración modelada con materiales absorbentes, se obtuvo una disminución de hasta $5 \mathrm{~dB}$ en la banda más crítica, lo que implica una disminución de $3 \mathrm{~dB}$ promedio, que corresponde al 50\% de disminución de la energía acústica recibida en 30 años de exposición y que para el caso estudiado aleja a la población de un daño médico-legal y con eso las eventuales indemnizaciones correspondientes. ${ }^{10}$

\section{DISCUSIÓN}

Es recomendable realizar audiometrías de ingreso al personal nuevo que se contrate, para detectar anomalias o susceptibilidades que puedan presentar estos trabajadores a la exposición a ruido. Los trabajadores de los puestos de trabajo evaluados, cuyas exposiciones a ruido resultaron con dosis de ruido diarias mayores a la Dosis de Acción de 0,5, deberán ingresar a un programa de vigi- lancia médica o mantenerlos si ya han ingresado, con el objeto de evaluar sus características de audición a través de exámenes audiométricos periódicos, para así controlar la evolución de la enfermedad a través del tiempo. Esta medida se debe hacer extensible al grupo homogéneo de trabajadores correspondientes a cada uno de los puestos de trabajo evaluados.

Para que la empresa logre controlar eficazmente los niveles de riesgo existentes, es necesario que implemente un programa de conservación auditiva interno, haciéndose cargo de:

a) Velar por el cumplimiento de las medidas de control de ruido propuestas, manteniendo los correspondientes registros.

b) Que a los trabajadores no solo se les entregue el elemento de protección auditiva adecuado, sino también instrucción respecto a su mantenimiento, uso correcto y permanente, deterioros debidos a causas mecánicas, suciedad, recambio oportuno y sobre el agente de riesgo contra el cual se les está protegiendo, manteniendo registros de estas actividades.

Finalmente, como medida de reducción de ruido complementaria, se sugiere el desacoplamiento mecánico de motores y sistemas rotatorios con el fin de evitar el puente acústico entre la maquinaria y la estructura soportante, evitando de esta forma la generación de ruido por vías aéreas y estructurales, producto de la vibración inducida. 
1. Chile. Ministerio de Salud. Decreto Supremo № 594/99. Reglamento sobre Condiciones Sanitarias y Ambientales Básicas en los Lugares de Trabajo, título IV, párrafo $3^{\circ}$, artículos 70-82.

2. Cárdenas JL, Ordinola J, Bravo J, Candia 0, Rojas A. Evaluación y control de dosis de ruido en trabajadores de la Empresa Puratos Chile S.A. [tesis] [en linea]. Santiago: UDLA; 2010 [consultado ene 2016] Disponible en: www. joseluiscardenas.com/tesis

3. Urnia JL. Prexor; Gestión para el control [presentación en línea] Santiago: Asociación Chilena de Seguridad; 2012 [consultado ene 2016]. Disponible en: www.uchile.cl/documentos/prexor_74636_7_0013.pdf

4. Ministerio de Salud Pública. Protocolo sobre normas mínimas para el desarrollo de programas de vigilancia de la pérdida auditiva por exposición a ruido en lugares de trabajo (Prexor): Santiago: Ministerio de Salud Pública; 2011. http:// web.minsal.cl/sites/default/files/files/protocolo_vigilancia_expuestos_a_ ruido_minsal.pdf.

5. Valenzuela JC, Sánchez M, Fontecilla Hernán. Diseño e Implementación de un estudio de diagnóstico de la exposición a ruido Laboral de los trabajadores de la región metropolitana en Chile. Santiago: Instituto de Salud Pública; 2012.

6. Chile. Ministerio de Salud. Decreto Supremo № 594/99, Condiciones sanitarias y Ambientales básicas en los lugares de trabajo. Ley Chile [en línea] (29-042000) [consultado ene 2016]. Disponible en: http://www.leychile.cl/Navegar? idNorma $=167766$

7. Ministerio de Salud. Protocolo de control y seguimiento a trabajadores expuestos a ruido y/o sordera laboral. Santiago; MINSAL; 1995.

8. International Organization for Standardization. ISO 1999:1990: Acoustics Determination of occupational noise exposure and estimation of noise induced hearing impairment [on line]. Genève: International Organization for Standardization;1990 [cited Jan 2016]. Available from: http://www.iso.org/iso/ catalogue_detail.htm?csnumber $=6759$

9. Canales AE. Informe técnico № 201301011558 sobre Evaluación de la Exposición Ocupacional a Ruido de la Empresa Acmanet, según D.S. N594/99 del Ministerio de Salud de Chile. Santiago: Asociación Chilena de Seguridad; 2013.

10. Miyara F. Estimación del riesgo auditivo por exposición a ruido según la Norma ISO 1999:1990. Buenos Aires: IRAM; 1999.

\section{Referencias complementarias:}

Bravo MT. Guia preventiva para los trabajadores expuestos a ruido. Santiago: ISP; 2012.

Canales A, Figueroa J, Cárdenas J. Caracterización acústica de transformadores AT, evaluación de impacto acústico en subestación Apoquindo y propuesta de metodologia de monitoreo de ruido en subestaciones de Chilectra S.A. [tesis]. Santiago: UDLA; 2009.

Cárdenas JL, Aguilera C, Evaluación del ambiente sonoro en área de instalación de equipos de perforación Hopper y equipo № 6 en ENAP Magallanes [tesis]. Santiago: UDLA; 2009.

Centro Privado de Investigaciones Acústicas. Catálogo Acústico [en línea]. Santiago: CPIA; 2010 [consultado ene 2016]. Disponible en: http://www.cpia.cl/index. php/catalogo-acustico.

Chile. Ministerio del Trabajo y de Previsión Social. Ley № 16.744; Establece normas sobre accidentes del trabajo y enfermedades profesionales. Santiago: Ministerio del Trabajo y Previsión Social; 1968.

Chile. Ministerio de Salud. Instructivo para la calificación y evaluación de las Enfermedades Profesionales del Reglamento D.S. № 109/1968 de la Ley №
16.744 [en línea]. Santiago: MINSAL; 2009 [consultado ene 2016]. Disponible en:http://163.247.55.110/PortalWEB/SST/circulares/Circular_B33-47_16-122009_MSalud.pdf

España. Ministerio de Empleo y Seguridad Social. Real decreto 286/2006, de 10 de marzo, sobre la protección de la salud y la seguridad de los trabajadores contra los riesgos relacionados con la exposición al ruido. BOE n 60 11/03/2006. Madrid: INSHT; 2006.

Instituto de Salud Pública de Chile. Guía técnica para la evaluación de los trabajadores expuestos a ruido y/o con sordera profesional [en línea]. Santiago: ISP; 2005 [consultado ene 2016]. Disponible en: www.ispch.cl/saludocupacional/ material_referencia

Instituto de Salud Pública de Chile. Instructivo para la aplicación del Decreto Supremo № 594/99, título IV, párrafo $3^{\circ}$, Agentes Físicos - Ruido [en línea]. Santiago: ISP; 2004 [consultado ene 2016]. Disponible en: www.ispch.cl/ salud_ocup/doc/INSTRUCTIV0_594.pdf

Instituto Nacional de Normalización. NCh 1331: Protectores auditivos; Requisitos y métodos de ensayo. Santiago: INN; 1999.

Instituto Nacional de Normalización. NCh 2502-1:1999-2000. Acústica-Descripción y Medición del Ruido Ambiental. Parte 1: Magnitudes Básicas y Procedimientos, Parte 2: Recolección de Datos Pertenecientes al Uso de Suelo, Parte 3: Aplicación a Limites de Ruido. Santiago: INN; 2000.

International Organization for Standardization. ISO 7029:2000 Acoustics -Statistical distribution of hearing thresholds as a function of age. Genève: International Organization for Standardization; 1990.

ISOVER. Manual de aislamiento en la industria. Madrid; ISOVER; 2007.

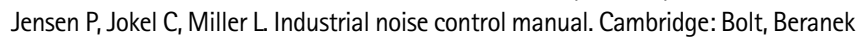
and Newman; 1978.

Martínez MC. Efectos del ruido por exposición laboral. Salud Trab [en línea] 1995 [consultado ene 2016] 3(2):93-101. Disponible en: www.ingenieroambiental. com/4014/eruido.pdf

National Institute for Occupational Safety and Health. Simulador Pérdida Auditiva Laboral; Guia de capacitación e instrucción. Atlanta: CDC-NIOSH; 2008. (DHHS-NIOSH Publicación Núm. 2008-119Sp) [consultado ene 2016]. Disponible en: http://www.cdc.gov/spanish/niosh/docs/2008-119_sp/pdfs/ 2008-119_sp.pdf

Orellana C. Diseño y determinación de parámetros acústicos de un material elaborado de caucho, y su posible aplicación en un área de trabajo de Codelco Chile División Salvador [tesis]. Valdivia: Universidad Austral de Chile; 2002.

Otárola MF, Otárola ZF, Finkelstein A. Ruido Laboral y su Impacto en Salud. Cienc Trab [en línea]. 2006 [consultado ene 2016];8(20):46-51.Disponible en: https://www.seguroscaracas.com/paginas/biblioteca_digital/PDF/1/ Documentos/Lesiones/Ruido\%20laboral\%20y\%20su\%20impacto\%20 en\%20salud.pdf

Pacheco G. Guia para la selección y control de protectores auditivos [tesis] [en línea]. Valdivia: UACH, 2006 [consultado ene 2016]. Disponible en: http:// cybertesis.uach.cl/tesis/uach/2006/bmfcip116g/doc/bmfcip116g.pdf

Reddy RK, Welch D, Thorne P, Ameratunga S. Hearing protection use in manufacturing Workers: A qualitative study. Noise Health. 2012;14(59):202-209.

Sánchez AM. Estrategia Frente a la Problemática del Ruido Ocupacional. Cienc trab. 2006;8(20):58-64.

Sánchez $\mathrm{M}$ et al. Guía para la selección y control De Protectores Auditivos según Resolución №1391-007 [en línea]. Santiago: ISP; 2007. www.ispch.cl/ elementos-de-proteccion-personal-epp 